\title{
An exact relation between free energy fluctuations and bond chaos in the Sherrington-Kirkpatrick model
}

\author{
T Aspelmeier \\ Max Planck Institute for Dynamics and Self Organization, 37073 Göttingen, \\ Germany
}

\begin{abstract}
Using a variant of the interpolating Hamiltonian technique, we show that there exists, in the Sherrington-Kirkpatrick spin glass, an exact connection between the sample-to-sample fluctuations of the free energy and bond chaos involving 2- and 4-replica overlaps between replicas with different but correlated bonds. This relation is used to derive an upper bound of the fluctuations.

PACS numbers: 75.50.Lk, 75.10.Nr
\end{abstract}

\section{Introduction}

Extreme value statistics is a very active field in current mathematical physics since the discovery of the Tracy-Widom distribution for the largest (or smallest) eigenvalue of a Gaussian random matrix, see [1] for an overview. The Tracy-Widom distribution is believed to consitute a new universality class for extreme values in addition to the three "classical" ones (Weibull, Gumbel, Fréchet). There are however many cases which do not fall in any of these four classes. One important example is the distribution of ground state energies in the Sherrington-Kirkpatrick model [2]. Despite tremendous numerical effort over the years [3, 4, 5, 6, 7, 8, 9, there is still no complete agreement as to what kind of distribution the ground states follow. Analytically, there is no theory (to the best of our knowledge) which would predict a particular limiting distribution for large system sizes $N$. Not even the width of the distribution is precisely known: the numerical simulations seem to suggest that the width scales as $N^{\mu}$ with $\mu \approx \frac{1}{4}$, and this is supported by some heuristic arguments [10, 4. Other arguments favour $\mu=\frac{1}{6}$ [11, 12]. (If this problem fell into the Tracy-Widom universality class, the width would scale as $N^{1 / 3}[\underline{6}$. This seems to be ruled out by the numerical results.)

In addition to the ground state energies and their sample-to-sample fluctuations one can also consider the sample-to-sample fluctuations of the free energy at a finite temperature within the spin glass phase. The natural expectation would be that in the low temperature phase these free energies fall into the same universality class as the ground state energies (although this has never been proved). However, the distribution of the free energies appears as inaccessible as the one of the ground state energies. Part of the difficulty lies in the fact that the variance of the distribution scales with a subextensive power of $N$. In order to calculate subextensive terms, it is usually necessary to go to higher than the leading order in the loop expansion of 
the spin glass problem. Due to the massless modes present throughout the spin glass phase this has so far been impossible in the Sherrington-Kirkpatrick model. For the finite-dimensional spin glass, this problem is not so severe and the fluctuations could be calculated 13, 14. They are however fundamentally different from the ones in the Sherrington-Kirkpatrick model, which we will be considering here.

In this paper we present a way which circumvents this obstacle by constructing an exact relation between the free energy fluctuations and bond chaos in spin glasses. This connection has been briefly described in [15] and we present the details of the calculation here. Using this relation, the width of the distribution can in principle be calculated by calculating chaos. A part of the necessary aspects of chaos has been calculated in [16, and the results from that paper will be sufficient to derive the upper bound $\mu \leq \frac{1}{4}$ here. For the full answer, it will be necessary to calculate more complicated objects such as simultaneous 4-replica overlaps. We will not be able to solve this formidable problem here.

This paper is organized as follows. In Sec. 2 we briefly review a few methods and results from the literature in order to compare them with our own theory later on. We derive the connection to bond chaos in Sec. 3. The fluctuations above and at the critical temperature, as well as the bound $\mu \leq \frac{1}{4}$ in the low temperature phase are calculated in Sec. 4. We end with a conclusion in Sec. 5.

\section{Above and at the critical temperature}

In this section we review a few methods and results above and at the critical temperature from the literature for completeness and for comparison with our own results later on.

Analytically, the free energy fluctuations of any disordered system can in principle be found with the replica method. Given the partition function $Z$ of a system of size $N$, it can easily be shown that a Taylor expansion of $\log \overline{Z^{n}}$ in powers of $n$ yields

$$
\log \overline{Z^{n}}=-n \beta F_{N}+\frac{n^{2}}{2} \Delta F_{N}^{2}+\cdots,
$$

where the overbar means the average over the disorder, $\beta=1 / k_{B} T$ is the inverse temperature, $F_{N}$ is the average free energy at system size $N$, and $\Delta F_{N}$ denotes its sample-to-sample fluctuations. The dots indicate higher order cumulants. Using the replica formalism, one can calculate $\overline{Z^{n}}$ for integer $n$ and try to continue the resulting expression to real (or, indeed, complex) $n$ and isolate the coefficient of the second order term which represents the fluctuations. In the case of the Ising spin glass this works very nicely above and at the critical temperature. It is straightforward to show with the standard replica formalism for the mean-field spin glass [17] that in the high temperature phase $(\beta<1)$, where the saddle point is replica symmetric and its Hessian has only strictly positive eigenvalues, the fluctuations are

$$
\beta^{2} \Delta F_{N}^{2}=-\frac{1}{2} \log \left(1-\beta^{2}\right)-\frac{\beta^{2}}{2}+\mathcal{O}(1 / N)
$$

11, 18. As the critical temperature $T_{c}$ is approached $\left(\beta \nearrow 1 / T_{c}=1\right)$, this expression diverges, which indicates that the fluctuations at the critical point must also diverge with $N$. A straightforward extension of the calculation in [18 shows that the fluctuations at the critical point are

$$
\beta^{2} \Delta F_{N}^{2}=\frac{1}{6} \log N+\mathcal{O}(1),
$$


which does indeed diverge as $N \rightarrow \infty$. As a check, we can rederive this result from Eq. (2) by isolating the divergent part, $-\frac{1}{2} \log (1-\beta)$, and replacing $1-\beta \sim \tau$ (where $\tau=\frac{T-T_{c}}{T_{c}}$ is the reduced temperature) by $x N^{-1 / 3}$. The variable $x=\tau N^{1 / 3}$ is the correct scaling combination in the critical region [18, 19]. Keeping $x$ fixed and letting $N$ tend to infinity in $-\frac{1}{2} \log \left(x N^{-1 / 3}\right)$ results in Eq. (3).

In the low temperature phase, the situation is much more complex and there are no reliable analytical results.

\section{Interpolating Hamiltonian}

In this section we will derive two different exact expressions for the fluctuations in terms of chaos using interpolating Hamiltonians. While the calculation presented here is in spirit similar to the one by Billoire [20, there is an important difference. Here, we do not interpolate between a big system and two small systems (see also 21]) but between two equally big systems. This may seem strange at first sight but is in fact the key to making any analytical progress on this particular problem.

\subsection{First route to chaos}

Consider the following interpolating Hamiltonian:

$$
\mathcal{H}_{t}=-\sqrt{\frac{1-t}{N}} \sum_{i<j} J_{i j} s_{i} s_{j}-\sqrt{\frac{t}{N}} \sum_{i<j} J_{i j}^{\prime} s_{i} s_{j}
$$

with $N$ Ising spins $s_{i}, 0 \leq t \leq 1$ and $J_{i j}, J_{i j}^{\prime}$ independent Gaussian random variables with unit variance. The parameter $t$ interpolates between one spin glass system $(t=0)$ and a statistically independent, but otherwise identical one at $t=1$. It is important to note that also for each other value of $t$ the Hamiltonian describes a normal spin glass, the coupling constants being $\sqrt{1-t} J_{i j}+\sqrt{t} J_{i j}^{\prime}$ which are Gaussian random variables with unit variance.

The partition function of this Hamiltonian is $Z_{t}=\operatorname{Tr} \exp \left(-\beta \mathcal{H}_{t}\right)$ and the free energy is $\beta F_{t}=-\log Z_{t}$. The sample-to-sample fluctuations of the free energy of the SK model can be obtained in the following way. Denoting the average over all coupling constants $J_{i j}$ and $J_{i j}^{\prime}$ (and later also $J_{i j}^{\prime \prime}$ and others) by $E \cdots$, we have

$$
\begin{aligned}
E\left(\log Z_{1}-\log Z_{0}\right)^{2} & =\beta^{2} E\left(F_{1}-F_{0}\right)^{2} \\
& =\beta^{2}\left(E F_{1}^{2}-2 E F_{1} F_{0}+E F_{0}^{2}\right) \\
& =2 \beta^{2}\left(\overline{F^{2}}-\bar{F}^{2}\right) \\
& =2 \beta^{2} \Delta F_{N}^{2} .
\end{aligned}
$$

The penultimate step follows from the fact that $E F_{1}^{2}=E F_{0}^{2}=: \overline{F^{2}}$ is the disorder average of the squared spin glass free energy and that the average $E F_{1} F_{0}=$ $\left(E F_{1}\right)\left(E F_{0}\right)=: \bar{F}^{2}$ factorizes into the square of the averaged free energy since the coupling constants in the two Hamiltonians $\mathcal{H}_{0}$ and $\mathcal{H}_{1}$ are independent. Using this formulation and the idea developed in [21] to represent $\log Z_{1}-\log Z_{0}$ by differentiating with respect to the interpolation parameter $t$ and immediately integrating again, the fluctuations can be written as

$$
2 \beta^{2} \Delta F_{N}^{2}=E\left(\log Z_{1}-\log Z_{0}\right)^{2}=\int_{0}^{1} d t \int_{0}^{1} d \tau E \frac{\partial}{\partial t} \log Z_{t} \frac{\partial}{\partial \tau} \log Z_{\tau} .(9)
$$


In Appendix A it is shown how to manipulate this expression in order to arrive at Eq. (A.14), which is repeated here for convenience. Note that this equation is exact.

$$
\begin{gathered}
E \frac{\partial}{\partial t} \log Z_{t} \frac{\partial}{\partial \tau} \log Z_{\tau}=\frac{N^{2} \beta^{4}}{16}\left(2-\frac{\sqrt{1-t} \sqrt{\tau}}{\sqrt{t} \sqrt{1-\tau}}-\frac{\sqrt{1-\tau} \sqrt{t}}{\sqrt{\tau} \sqrt{1-t}}\right) E\left\langle\left(q_{13}^{2}-q_{14}^{2}\right)\left(q_{13}^{2}-q_{23}^{2}\right)\right\rangle \\
+\frac{N \beta^{2}}{4 \sqrt{t \tau}}\left(E\left\langle q_{13}^{2}\right\rangle-\frac{1}{N}\right)
\end{gathered}
$$

The symbols $q_{a b}$ are overlaps between independent replicas with different interpolation parameters,

$$
q_{a b}(t, \tau)=\frac{1}{N} \sum_{i} s_{i}^{a, t} s_{i}^{b, \tau} .
$$

In Eq. (10) replicas 1 and 2 have parameter $t$ and replicas 3 and 4 have parameter $\tau$. The angular brackets $\langle\cdots\rangle$ denote the thermal average of a system of independent replicas with the appropriate interpolation parameters.

We have thus established a connection between the fluctuations and the overlap between replicas with different interpolation parameters. The last important step is to realize that for any given value of $t, \mathcal{H}_{t}$ represents a normal mean-field spin glass with Gaussian couplings just like any other. The overlap $q_{13}$ between two replicas with different interpolation parameters is therefore an overlap between two normal spin glasses with identical bonds (if $t=\tau$ ), uncorrelated bonds (if $t=0, \tau=1$ or vice versa) or related, but not equal bonds (for anything in between). This immediately shows the connection to chaos in spin glasses. Chaos concerns the question how the equilibrium states of two initially equal systems are related when a small perturbation is applied to one of them, e.g. a small change of temperature (temperature chaos) or a perturbation of the bonds (bond chaos). When there is chaos, the equilibrium states are completely unrelated and the overlap is 0 (in the thermodynamic limit), no matter how small the perturbation. In our case, we are dealing with bond chaos.

Let $t$ and $\tau$ be given and let the coupling constants of $\mathcal{H}_{t}$ be the reference configuration of bonds: $K_{i j}^{0}:=\sqrt{1-t} J_{i j}+\sqrt{t} J_{i j}^{\prime}$. The coupling constants belonging to $\mathcal{H}_{\tau}$ are $K_{i j}=\sqrt{1-\tau} J_{i j}+\sqrt{\tau} J_{i j}^{\prime}$. Since the $J_{i j}$ and $J_{i j}^{\prime}$ are Gaussian random variables, so are $K_{i j}^{0}$ and $K_{i j}$ (also with unit variance). Their correlation is $E_{J, J^{\prime}} K_{i j}^{0} K_{i j}=$ $\sqrt{1-t} \sqrt{1-\tau}+\sqrt{t \tau}$. Instead of using $J_{i j}$ and $J_{i j}^{\prime}$ as the basic independent random variables one could also use $K_{i j}^{0}$, introduce new Gaussian random variables $K_{i j}^{\prime}$ and take $K_{i j}^{0}$ and $K_{i j}^{\prime}$ as the building blocks of the random variables. We can then write the bonds pertaining to $\mathcal{H}_{\tau}$ as

$$
K_{i j}(\epsilon)=\frac{K_{i j}^{0}}{\sqrt{1+\epsilon^{2}}}+\frac{\epsilon K_{i j}^{\prime}}{\sqrt{1+\epsilon^{2}}},
$$

such that the correlation between $K_{i j}^{0}$ and $K_{i j}(\epsilon)$ is $E K_{i j}^{0} K_{i j}(\epsilon)=\frac{1}{\sqrt{1+\epsilon^{2}}}$. In order that the bonds $K_{i j}(\epsilon)$ are statistically equivalent to the original bonds of $\mathcal{H}_{\tau}$, the correlation must be equal to the correlation obtained before, so

$$
\frac{1}{\sqrt{1+\epsilon^{2}}}=\sqrt{1-t} \sqrt{1-\tau}+\sqrt{t \tau}
$$

Thus we see that the disorder average of the overlap $q_{13}(t, \tau)$ is only a function of the "distance" $\epsilon$ of the coupling constants, i.e. $E\left\langle q_{13}^{2}(t, \tau)\right\rangle=E\left\langle q_{13}^{2}(\epsilon)\right\rangle$ is only a function of $\epsilon$, not of $t$ and $\tau$ indepently. The same applies of course for products of overlaps 
such as $E\left\langle q_{13}^{2}(t, \tau) q_{23}^{2}(t, \tau)\right\rangle=E\left\langle q_{13}^{2}(\epsilon) q_{23}^{2}(\epsilon)\right\rangle$. The distance $\epsilon$ varies between 0 and $\infty$.

In order to obtain the fluctuations, we must integrate Eq. (28) over $t$ and $\tau$, according to Eq. (91). But since the overlaps only depend on $\epsilon$, it is useful to make a variable substitution and go over to $\epsilon$ and $z:=\sqrt{1+\epsilon^{2}} \sqrt{\tau}$. We first note that the integral $\int_{0}^{1} d t \int_{0}^{1} d \tau \bullet$ can be restricted to the range $\tau \leq t$ due to symmetry, provided a factor of 2 is inserted. We can then make the substitution and obtain

$\beta^{2} \Delta F_{N}^{2}=-\frac{N^{2} \beta^{4}}{16} \int_{0}^{\infty} d \epsilon f_{1}(\epsilon) E\left\langle\left(q_{13}^{2}-q_{14}^{2}\right)\left(q_{13}^{2}-q_{23}^{2}\right)\right\rangle+\frac{N \beta^{2}}{4} \int_{0}^{\infty} d \epsilon g_{1}(\epsilon)\left(E\left\langle q_{13}^{2}\right\rangle-\frac{1}{N}\right)$

where

$$
\begin{aligned}
& f_{1}(\epsilon)=\int_{0}^{1} d z \mathcal{J} \times\left(\frac{\sqrt{1-t} \sqrt{\tau}}{\sqrt{t} \sqrt{1-\tau}}+\frac{\sqrt{1-\tau} \sqrt{t}}{\sqrt{\tau} \sqrt{1-t}}-2\right), \\
& g_{1}(\epsilon)=\int_{0}^{1} d z \mathcal{J} \times \frac{1}{\sqrt{t \tau}}
\end{aligned}
$$

with the Jacobian

$$
\mathcal{J}=\frac{4 z}{\left(1+\epsilon^{2}\right)^{4}}\left(\epsilon \sqrt{1+\epsilon^{2}-z^{2}}+z\right)\left(\sqrt{1+\epsilon^{2}-z^{2}}-\epsilon z\right) .
$$

The old variables $t$ and $\tau$, expressed in terms of the new ones, are

$$
\begin{aligned}
& t=\left(\frac{\epsilon \sqrt{1+\epsilon^{2}-z^{2}}+z}{1+\epsilon^{2}}\right)^{2}, \\
& \tau=\frac{z^{2}}{1+\epsilon^{2}} .
\end{aligned}
$$

The integrals in Eqs. (15) and (16) can be evaluated explicitly and we find

$$
\begin{aligned}
& f_{1}(\epsilon)=\frac{4 \epsilon^{2}}{\left(1+\epsilon^{2}\right)^{2}} \arcsin \frac{1}{\sqrt{1+\epsilon^{2}}} \\
& g_{1}(\epsilon)=\frac{2}{\left(1+\epsilon^{2}\right)^{3 / 2}} \arcsin \frac{1}{\sqrt{1+\epsilon^{2}}} .
\end{aligned}
$$

Eq. (14) is our first important result. It is exact and connects the fluctuations with bond chaos. If it were possible to calculate bond chaos (and it was shown in [16] that at least for the 2-replica overlaps it is possible), the flucutations follow immediately since the functions $f_{1}(\epsilon)$ and $g_{1}(\epsilon)$ are "harmless" (Eqs. (20) and (21)). Note that $f_{1}(\epsilon)$ and $g_{1}(\epsilon)$ are nonnegative and $\left\langle\left(q_{13}^{2}-q_{14}^{2}\right)\left(q_{13}^{2}-q_{23}^{2}\right)\right\rangle$ is also nonnegative (this is shown in Appendix A). The first term in Eq. (14) is therefore negative. Hence the second term is an upper bound for the fluctuations.

\subsection{Second route to chaos}

There is another way to represent the fluctuations with interpolating Hamiltonians than Eq. (8) which will lead to a second expression for the fluctuations. Introducing the Hamilontian $\mathcal{H}_{t}^{\prime}$ defined by

$$
\mathcal{H}_{t}^{\prime}=-\sqrt{\frac{1-t}{N}} \sum_{i<j} J_{i j} s_{i} s_{j}-\sqrt{\frac{t}{N}} \sum_{i<j} J_{i j}^{\prime \prime} s_{i} s_{j}
$$


which only differs from $\mathcal{H}_{t}$ by the second set of coupling constants $J_{i j}^{\prime \prime}$ which are again independent Gaussian random variables with unit variance, we can write

$$
\begin{aligned}
E\left(\log Z_{1}-\log Z_{0}\right)\left(\log Z_{1}^{\prime}-\log Z_{0}^{\prime}\right) & =\beta^{2} E\left(F_{1}-F_{0}\right)\left(F_{1}^{\prime}-F_{0}^{\prime}\right) \\
& =\beta^{2} E\left(F_{1} F_{1}^{\prime}-F_{1} F_{0}^{\prime}-F_{0} F_{1}^{\prime}+F_{0} F_{0}^{\prime}\right) \\
& =\beta^{2}\left(\overline{F^{2}}-\bar{F}^{2}\right) \\
& =\beta^{2} \Delta F_{N}^{2},
\end{aligned}
$$

where $Z_{t}^{\prime}$ and $F_{t}^{\prime}$ are the partition function and the free energy pertaining to $\mathcal{H}_{t}^{\prime}$. The fluctuations can be represented by a double integral, as above,

$$
\beta^{2} \Delta F_{N}^{2}=\int_{0}^{1} d t \int_{0}^{1} d \tau E \frac{\partial}{\partial t} \log Z_{t} \frac{\partial}{\partial \tau} \log Z_{\tau}^{\prime} .
$$

Proceeding precisely as above and in Appendix Appendix A, we get

$$
\begin{gathered}
E \frac{\partial}{\partial t} \log Z_{t} \frac{\partial}{\partial \tau} \log Z_{\tau}^{\prime}=\frac{N^{2} \beta^{4}}{16} E\left\langle\left(q_{13}^{2}-q_{14}^{2}\right)\left(q_{13}^{2}-q_{23}^{2}\right)\right\rangle \\
+\frac{N \beta^{2}}{8 \sqrt{1-t} \sqrt{1-\tau}}\left(E\left\langle q_{13}^{2}\right\rangle-\frac{1}{N}\right) .
\end{gathered}
$$

Replicas 1 and 2 have Hamiltonian $\mathcal{H}_{t}$ and replicas 3 and 4 have $\mathcal{H}_{\tau}^{\prime}$.

Integrating over $t$ and $\tau$ gives us the fluctuations, and again the overlaps do not depend on $t$ and $\tau$ separately but only on the distance $\epsilon$. The distance is here not given by Eq. (13) but is slightly different due to the independence of the $J^{\prime}$ s and $J^{\prime \prime}$ s. Arguing similarly as above, $\epsilon$ is found to be related to $t$ and $\tau$ by

$$
\frac{1}{\sqrt{1+\epsilon^{2}}}=\sqrt{1-t} \sqrt{1-\tau} \text {. }
$$

Making a change of variables to eliminate $\tau$ in favour of $\epsilon$ yields

$$
\begin{aligned}
\beta^{2} \Delta F_{N}^{2}= & \frac{N^{2} \beta^{4}}{16} \int_{0}^{\infty} d \epsilon \int_{0}^{\epsilon^{2} /\left(1+\epsilon^{2}\right)} d t \frac{2 \epsilon}{(1-t)\left(1+\epsilon^{2}\right)^{2}} E\left\langle\left(q_{13}^{2}-q_{14}^{2}\right)\left(q_{13}^{2}-q_{23}^{2}\right)\right\rangle \\
& +\frac{N \beta^{2}}{8} \int_{0}^{\infty} d \epsilon \int_{0}^{\epsilon^{2} /\left(1+\epsilon^{2}\right)} d t \frac{2 \epsilon}{(1-t)\left(1+\epsilon^{2}\right)^{2}} \sqrt{1+\epsilon^{2}}\left(E\left\langle q_{13}^{2}\right\rangle-\frac{1}{N}\right),
\end{aligned}
$$

such that

$\beta^{2} \Delta F_{N}^{2}=\frac{N^{2} \beta^{4}}{16} \int_{0}^{\infty} d \epsilon f_{2}(\epsilon) E\left\langle\left(q_{13}^{2}-q_{14}^{2}\right)\left(q_{13}^{2}-q_{23}^{2}\right)\right\rangle+\frac{N \beta^{2}}{4} \int_{0}^{\infty} d \epsilon g_{2}(\epsilon)\left(E\left\langle q_{13}^{2}\right\rangle-\frac{1}{N}\right)$.

This is the second result for the fluctuations. It has precisely the same structure as Eq. (14). The only difference are the weight functions under the integrals, which are given by

$$
\begin{aligned}
& f_{2}(\epsilon)=\frac{2 \epsilon \log \left(1+\epsilon^{2}\right)}{\left(1+\epsilon^{2}\right)^{2}} \\
& g_{2}(\epsilon)=\frac{\epsilon \log \left(1+\epsilon^{2}\right)}{\left(1+\epsilon^{2}\right)^{3 / 2}},
\end{aligned}
$$

and the sign of the first term, which here is positive. 


\section{Calculation of the fluctuations}

Having established the connection to chaos, we can proceed to calculate the fluctuations by calculating $E\left\langle q_{13}^{2}\right\rangle$ and $E\left\langle\left(q_{13}^{2}-q_{14}^{2}\right)\left(q_{13}^{2}-q_{23}^{2}\right)\right\rangle$. The former can be accomplished by taking the bond averaged probability distribution $P_{\epsilon}(q)$ of the overlap $q$ for bond chaos, which has been calculated in [16]. Averages taken with this probability distribution will be denoted by $[\cdots]_{0}$. The latter is more difficult and will be postponed to a later publication. It requires the joint probability distributions $P_{\epsilon}^{123}\left(q_{13}, q_{23}\right)$ and $P_{\epsilon}^{1234}\left(q_{13}, q_{24}\right)$. However, above and at the critical temperature, there is no replica symmetry breaking, hence these probability distributions factorize into $P_{\epsilon}^{123}\left(q_{13}, q_{23}\right)=P_{\epsilon}\left(q_{13}\right) P_{\epsilon}\left(q_{23}\right)$ and $P_{\epsilon}^{1234}\left(q_{14}, q_{23}\right)=P_{\epsilon}\left(q_{14}\right) P_{\epsilon}\left(q_{23}\right)$ such that

$$
E\left\langle\left(q_{13}^{2}-q_{14}^{2}\right)\left(q_{13}^{2}-q_{23}^{2}\right)\right\rangle=\left[q^{4}\right]_{0}-\left[q^{2}\right]_{0}^{2} .
$$

We will be able to calculate this. Below the critical temperature, on the other hand, we will have to content ourselves with an upper bound of the fluctuations which is given by the second integral in Eq. (14).

\subsection{Above the critical temperature}

The nonnormalised probability distribution of the overlap $q$ for bond chaos, $R_{\epsilon}(q)$, above the critical temperature is [16]

$$
R_{\epsilon}^{0}(q)=e^{-N\left(\frac{q^{2}}{2} h(\epsilon)+\mathcal{O}\left(q^{4}\right)\right)}
$$

with $h(\epsilon)=1-\frac{\beta^{2}}{\sqrt{1+\epsilon^{2}}}$.

For large $N$, we can easily calculate $\left[q^{2}\right]_{0}$ and $\left[q^{4}\right]_{0}$ via steepest descents. At leading order, the terms of order $q^{4}$ and higher in the exponent do not contribute. Defining $q_{n}:=\int_{0}^{\infty} d q q^{n} R_{\epsilon}(q)$ (the upper bound may be set to $\infty$ as this only introduces exponentially small errors), we get

$$
q_{n}=\frac{1}{2}\left(\frac{N}{2} h(\epsilon)\right)^{-(n+1) / 2} \Gamma\left(\frac{n+1}{2}\right)
$$

such that

$$
\begin{aligned}
& {\left[q^{2}\right]_{0}=\frac{q_{2}}{q_{0}}=\frac{1}{N h(\epsilon)},} \\
& {\left[q^{4}\right]_{0}=\frac{q_{4}}{q_{0}}=\frac{3}{N^{2} h^{2}(\epsilon)} .}
\end{aligned}
$$

This allows us to write down two equations for the fluctuations from our two routes to chaos, Eqs. (14) and (31), namely

$$
\begin{aligned}
\beta^{2} \Delta F_{N}^{2} & =-\frac{\beta^{4}}{8} \int_{0}^{\infty} d \epsilon \frac{f_{1}(\epsilon)}{h^{2}(\epsilon)}+\frac{\beta^{2}}{4} \int_{0}^{\infty} d \epsilon g_{1}(\epsilon)\left(\frac{1}{h(\epsilon)}-1\right) \\
& =\frac{\beta^{4}}{8} \int_{0}^{\infty} d \epsilon \frac{f_{2}(\epsilon)}{h^{2}(\epsilon)}+\frac{\beta^{2}}{4} \int_{0}^{\infty} d \epsilon g_{2}(\epsilon)\left(\frac{1}{h(\epsilon)}-1\right) .
\end{aligned}
$$

The second of these expressions can be evaluated explictly, with the result

$$
\beta^{2} \Delta F_{N}^{2}=-\frac{1}{2} \log \left(1-\beta^{2}\right)-\frac{\beta^{2}}{2},
$$

in accordance with Eq. (2). The author is currently unable to calculate the integrals in Eq. (39) but numerical checks show that they give precisely the same result. 


\subsection{At the critical temperature}

The nonnormalised probability distribution $R_{\epsilon}(q)$ precisely at the critical temperature is given by [16]

$$
R_{\epsilon}(q)=\left\{\begin{array}{ll}
e^{-N w q^{3} / 6} & \epsilon \ll N^{-1 / 6} \\
e^{-N q^{2} h(\epsilon) / 2} & N^{-1 / 6} \ll \epsilon
\end{array} .\right.
$$

Define as before $q_{n}=\int_{0}^{\infty} d q q^{n} R_{\epsilon}(q)$. Then we find

$$
q_{n}=\left\{\begin{array}{ll}
\frac{1}{3}\left(\frac{N w}{6}\right)^{-(n+1) / 3} \Gamma\left(\frac{n+1}{3}\right) & \epsilon \ll N^{-1 / 6} \\
\frac{1}{2}\left(\frac{N}{2} h(\epsilon)\right)^{-(n+1) / 2} \Gamma\left(\frac{n+1}{2}\right) & N^{-1 / 6} \ll \epsilon
\end{array},\right.
$$

such that

$$
\left[q^{2}\right]_{0}=\frac{q_{2}}{q_{0}}= \begin{cases}\left(\frac{N w}{6}\right)^{-2 / 3} \frac{1}{\Gamma\left(\frac{1}{3}\right)} & \epsilon \ll N^{-1 / 6} \\ \left(\frac{N}{2} h(\epsilon)\right)^{-1} \frac{\Gamma\left(\frac{3}{2}\right)}{\Gamma\left(\frac{1}{2}\right)} & N^{-1 / 6} \ll \epsilon\end{cases}
$$

and

$$
\left[q^{4}\right]_{0}=\frac{q_{4}}{q_{0}}=\left\{\begin{array}{ll}
\left(\frac{N w}{6}\right)^{-4 / 3} \frac{\Gamma\left(\frac{5}{3}\right)}{\Gamma\left(\frac{1}{3}\right)} & \epsilon \ll N^{-1 / 6} \\
\left(\frac{N}{2} h(\epsilon)\right)^{-2} \frac{\Gamma\left(\frac{5}{2}\right)}{\Gamma\left(\frac{1}{2}\right)} & N^{-1 / 6} \ll \epsilon
\end{array} .\right.
$$

Now we can evaluate Eq. (31). Plugging $\left[q^{2}\right]_{0}$ into the second term of that equation yields a constant of order 1 which is not of interest and will therefore not be calculated explicitly. The first term, however, is important. Splitting the integral into two parts we get asymptotically

$$
\begin{gathered}
\frac{N^{2} \beta^{4}}{16} \int_{0}^{\infty} d \epsilon f_{2}(\epsilon)\left(\left[q^{4}\right]_{0}-\left[q^{2}\right]_{0}^{2}\right)=\frac{N^{2} \beta^{4}}{16} \int_{0}^{N^{-1 / 6}} d \epsilon f_{2}(\epsilon) \frac{\Gamma\left(\frac{5}{3}\right) \Gamma\left(\frac{1}{3}\right)-1}{\Gamma^{2}\left(\frac{1}{3}\right)}\left(\frac{N w}{6}\right)^{-4 / 3}(46) \\
+\frac{N^{2} \beta^{4}}{16} \int_{N^{-1 / 6}}^{\infty} d \epsilon f_{2}(\epsilon) \frac{1}{2}\left(\frac{N}{2} h(\epsilon)\right)^{-2}
\end{gathered}
$$

The first of these integrals yield a constant (independent of $N$ ). The second one, however, gives a logarithm at the lower bound such that we get (with $f_{2}(\epsilon)=$ $2 \epsilon^{3}+\mathcal{O}\left(\epsilon^{5}\right), h(\epsilon)=\epsilon^{2} / 2+\mathcal{O}\left(\epsilon^{4}\right)$ and $\beta=1$ as we are at the critical point)

$$
\beta^{2} \Delta F_{N}^{2}=\frac{1}{6} \log N+\mathcal{O}(1) .
$$

This is precisely the known result.

It is interesting to note that we would not have been able to obtain this result so easily from our first route to chaos, Eq. (14), as both integrals in that expression grow with some power of $N$, and only their difference cancels out the leading behaviour and leaves a logarithmic divergence. In order to actually calculate this, we would need subleading corrections to the integrals, which would be very hard to obtain indeed.

\subsection{Below the critical temperature}

Now we turn to the low temperature phase. We will not be able here to solve the complete problem since $P_{\epsilon}^{123}\left(q_{13}, q_{23}\right)$ and $P_{\epsilon}^{1234}\left(q_{14}, q_{23}\right)$ do not factorize in the symmetry breaking phase. In [22, 23] it has been shown how to break down these probability distributions but the results only apply for $\epsilon=0$. Instead, we focus on the 
second term in Eq. (14) since it only requires $P_{\epsilon}\left(q_{13}\right)$ and provides an upper bound for the fluctuations.

From [16] we get the nonnormalised probability distribution of $q$ in the low temperature phase, which is

$$
R_{\epsilon}(q)=\left\{\begin{array}{ll}
\hat{\theta}\left(q-q_{\mathrm{EA}}\right) & \epsilon \ll N^{-1 / 2} \\
e^{-N c_{1} \epsilon^{2} q^{3}} & N^{-1 / 2} \ll \epsilon \ll N^{-1 / 5} \\
e^{-N c_{2} \epsilon^{3} q^{2}} & N^{-1 / 5} \ll \epsilon \leq \epsilon_{0} \\
e^{-N f(\epsilon) q^{2}} & \epsilon_{0}<\epsilon
\end{array},\right.
$$

where

$$
\hat{\theta}(x)= \begin{cases}1 & x<0 \\ e^{-N c_{0} x^{3}} & x>0\end{cases}
$$

with some (unimportant) positive constant $c_{0}$ and $q_{\mathrm{EA}}$ is the Edwards-Anderson order parameter, such that

$$
q_{n}= \begin{cases}\frac{q_{\mathrm{EA}}^{n+1}}{n+1} & \epsilon \ll N^{-1 / 2} \\ \frac{1}{3}\left(N c_{1} \epsilon^{2}\right)^{-(n+1) / 3} \Gamma\left(\frac{n+1}{3}\right) & N^{-1 / 2} \ll \epsilon \ll N^{-1 / 5} \\ \frac{1}{2}\left(N c_{2} \epsilon^{3}\right)^{-(n+1) / 2} \Gamma\left(\frac{n+1}{2}\right) & N^{-1 / 5} \ll \epsilon \leq \epsilon_{0} \\ \frac{1}{2}(N f(\epsilon))^{-(n+1) / 2} \Gamma\left(\frac{n+1}{2}\right) & \epsilon_{0}<\epsilon\end{cases}
$$

and

$$
\left[q^{2}\right]_{0} \propto\left\{\begin{array}{ll}
\text { const. } & \epsilon \ll N^{-1 / 2} \\
\left(N \epsilon^{2}\right)^{-2 / 3} & N^{-1 / 2} \ll \epsilon \ll N^{-1 / 5} \\
\left(N \epsilon^{3}\right)^{-1} & N^{-1 / 5} \ll \epsilon \leq \epsilon_{0} \\
(N f(\epsilon))^{-1} & \epsilon_{0}<\epsilon
\end{array} .\right.
$$

Note the discussion in [16] about why the probability distribution for $\epsilon \ll N^{-1 / 2}$ does not coincide with the true distribution for the Sherrington-Kirkpatrick model. However, this discrepancy only changes the value of $\left[q^{2}\right]_{0}$ for small $\epsilon$. It does not change the qualitative behaviour of $\left[q^{2}\right]_{0}$ as a function of $\epsilon$.

We can estimate the integral $\frac{N \beta^{2}}{4} \int_{0}^{\infty} d \epsilon g_{1}(\epsilon)\left(E\left\langle q_{13}^{2}\right\rangle-\frac{1}{N}\right)$ from Eq. (14) by first neglecting the $1 / N$-term under the integral as we are only interested in the leading behaviour. We can also neglect the contribution of the integration from $\epsilon_{0}$ to $\infty$ since it will only be of order 1 . We also note that we can combine the regions $\epsilon \ll N^{-1 / 2}$ and $N^{-1 / 2} \ll \epsilon \ll N^{-1 / 5}$ by writing $\left[q^{2}\right]_{0}=\mathcal{F}\left(N^{1 / 2} \epsilon\right)$ with a scaling function $\mathcal{F}(x)$ with the properties $\mathcal{F}(x) \rightarrow$ const. $(x \rightarrow 0)$ and $\mathcal{F}(x) \sim x^{-4 / 3}(x \rightarrow \infty)$. We then obtain for the first part of the integral (expanding the function $g_{1}(\epsilon)$ for small $\epsilon$ )

$$
\begin{aligned}
& \frac{N \beta^{2}}{4} \int_{0}^{N^{-1 / 5}} d \epsilon g_{1}(\epsilon)\left[q^{2}\right]_{0} \approx \frac{N \beta^{2}}{4} \pi \int_{0}^{N^{-1 / 5}} d \epsilon \mathcal{F}\left(N^{1 / 2} \epsilon\right) \\
& =N^{1 / 2} \frac{\beta^{2}}{4} \pi \int_{0}^{N^{3 / 10}} d x \mathcal{F}(x) \sim N^{1 / 2} .
\end{aligned}
$$

The next part of the integral is

$$
\frac{N \beta^{2}}{4} \int_{N^{-1 / 5}}^{\epsilon_{0}} d \epsilon g_{1}(\epsilon)\left[q^{2}\right]_{0} \sim \frac{N \beta^{2}}{4} \pi \int_{N^{-1 / 5}}^{\epsilon_{0}} \frac{1}{N \epsilon^{3}} \sim N^{2 / 5} .
$$

This contribution is smaller than the one we just had and may be neglected.

The final answer for the fluctuations in the low temperature phase is therefore

$$
\beta^{2} \Delta F_{N}^{2} \leq \text { const. } \times N^{1 / 2},
$$


i.e. we get the upper bound

$$
\mu \leq \frac{1}{4}
$$

\section{Conclusion}

We have shown that the free energy fluctuations in the Sherrington-Kirkpatrick model can be expressed in two different ways in terms of bond chaos, Eqs. (14) and (31), both of which are exact. The first formulation consists of a difference of two positive terms while the second is a sum of positive terms. We have derived an upper bound of the fluctuations using the first formulation, resulting in $\mu \leq \frac{1}{4}$. In the future, the second formulation will be more useful because it allows direct access to the fluctuations when 4-replica overlaps are calculated, either numerically or analytically, since it is easy to see that the second integral in Eq. (31) is subdominant and only the first integral needs to be evaluated in order to obtain the leading behaviour of the fluctuations.

\section{Acknowledgments}

I would like to thank M. Goethe, A. Braun and M.A. Moore for many useful discussions.

\section{Appendix A. Evaluation of the interpolating Hamiltonians}

In this appendix we show the details of the derivation of the connection to chaos. The partial derivatives in Eq. (9) evaluate to

$$
\begin{aligned}
\frac{\partial}{\partial t} \log Z_{t} & =\frac{1}{Z_{t}} \operatorname{Tr}\left(\frac{\beta}{2 \sqrt{t} \sqrt{N}} \sum_{i<j} J_{i j}^{\prime} s_{i} s_{j}-\frac{\beta}{2 \sqrt{1-t} \sqrt{N}} \sum_{i<j} J_{i j} s_{i} s_{j}\right) \exp \left(-\beta \mathcal{H}_{t}\right) \\
& =\frac{1}{2 t} \sum_{i<j} J_{i j}^{\prime} \frac{\partial \log Z_{t}}{\partial J_{i j}^{\prime}}-\frac{1}{2(1-t)} \sum_{i<j} J_{i j} \frac{\partial \log Z_{t}}{\partial J_{i j}}
\end{aligned}
$$

It remains to deal with the average over the disorder in

$$
\begin{aligned}
E \frac{\partial}{\partial t} \log Z_{t} \frac{\partial}{\partial \tau} \log Z_{\tau}= & E \sum_{i<j, k<l}\left(\frac{1}{2 t} J_{i j}^{\prime} \frac{\partial \log Z_{t}}{\partial J_{i j}^{\prime}}-\frac{1}{2(1-t)} J_{i j} \frac{\partial \log Z_{t}}{\partial J_{i j}}\right) \\
& \times\left(\frac{1}{2 \tau} J_{k l}^{\prime} \frac{\partial \log Z_{\tau}}{\partial J_{k l}^{\prime}}-\frac{1}{2(1-\tau)} J_{k l} \frac{\partial \log Z_{\tau}}{\partial J_{k l}}\right) \cdot(\mathrm{A} .3)
\end{aligned}
$$

Let's look at the first term of the product under the sum, $E \frac{1}{4 t \tau} J_{i j}^{\prime} J_{k l}^{\prime} \frac{\partial \log Z_{t}}{\partial J_{i j}^{\prime}} \frac{\partial \log Z_{\tau}}{\partial J_{k l}^{\prime}}$. We can integrate by parts with respect to, say, $J_{i j}^{\prime}$ (a standard trick 21]) in the form

$$
\begin{aligned}
E J_{i j}^{\prime} J_{k l}^{\prime} \bullet & =\int \cdots d J_{i j}^{\prime} e^{-J_{i j}^{\prime 2} / 2} \cdots J_{i j}^{\prime} J_{k l}^{\prime} \bullet \\
& =\int \cdots d J_{i j}^{\prime} e^{-J_{i j}^{\prime 2} / 2} \cdots \frac{\partial}{\partial J_{i j}^{\prime}} J_{k l}^{\prime} \bullet=E \frac{\partial}{\partial J_{i j}^{\prime}} J_{k l}^{\prime} \bullet
\end{aligned}
$$

where the $\bullet$ stands symbollically for any function of the $J_{i j}$ and $J_{i j}^{\prime}$. The derivative can be moved to the right using the product rule so

$$
E J_{i j}^{\prime} J_{k l}^{\prime} \bullet=E\left(\delta_{(i j),(k l)}+J_{k l}^{\prime} \frac{\partial}{\partial J_{i j}^{\prime}}\right) \bullet .
$$


Here, the second term can once again be treated by integration by parts, this time with respect to $J_{k l}^{\prime}$. The result is

$$
E J_{i j}^{\prime} J_{k l}^{\prime} \bullet=E\left(\delta_{(i j),(k l)}+\frac{\partial}{\partial J_{k l}^{\prime}} \frac{\partial}{\partial J_{i j}^{\prime}}\right) \bullet .
$$

The same procedure can be applied to the remaining terms in Eq. (A.3), with the difference that the terms that mix $J_{\mathrm{S}}$ and $J^{\prime} \mathrm{s}$ do not have the $\delta_{(i j),(k l)}$, resulting in

$$
\begin{gathered}
E \frac{\partial}{\partial t} \log Z_{t} \frac{\partial}{\partial \tau} \log Z_{\tau}=E \sum_{i<j, k<l}\left[\frac{1}{4 t \tau} \frac{\partial}{\partial J_{k l}^{\prime}} \frac{\partial}{\partial J_{i j}^{\prime}} \frac{\partial \log Z_{t}}{\partial J_{i j}^{\prime}} \frac{\partial \log Z_{\tau}}{\partial J_{k l}^{\prime}}-\frac{1}{4 t(1-\tau)} \frac{\partial}{\partial J_{k l}} \frac{\partial}{\partial J_{i j}^{\prime}} \frac{\partial \log Z_{t}}{\partial J_{i j}^{\prime}} \frac{\partial \log Z_{\tau}}{\partial J_{k l}}\right. \\
\left.-\frac{1}{4(1-t) \tau} \frac{\partial}{\partial J_{k l}^{\prime}} \frac{\partial}{\partial J_{i j}} \frac{\partial \log Z_{t}}{\partial J_{i j}} \frac{\partial \log Z_{\tau}}{\partial J_{k l}^{\prime}}+\frac{1}{4(1-t)(1-\tau)} \frac{\partial}{\partial J_{k l}} \frac{\partial}{\partial J_{i j}} \frac{\partial \log Z_{t}}{\partial J_{i j}} \frac{\partial \log Z_{\tau}}{\partial J_{k l}}\right] \\
+\frac{1}{4 t \tau} \sum_{i<j} E \frac{\partial \log Z_{t}}{\partial J_{i j}^{\prime}} \frac{\partial \log Z_{\tau}}{\partial J_{i j}^{\prime}}+\frac{1}{4(1-t)(1-\tau)} \sum_{i<j} E \frac{\partial \log Z_{t}}{\partial J_{i j}} \frac{\partial \log Z_{\tau}}{\partial J_{i j}}
\end{gathered}
$$

The derivatives which appear in this expression are related to spin averages. One finds for example

$$
\frac{\partial \log Z_{t}}{\partial J_{i j}}=\frac{\beta}{\sqrt{N}} \sqrt{1-t}\left\langle s_{i} s_{j}\right\rangle_{t}
$$

where $\langle\cdots\rangle_{t}$ stands for the thermal average, to be taken with the interpolation parameter set to $t$. Similarly, for two derivatives, one obtains for instance

$$
\frac{\partial^{2} \log Z_{t}}{\partial J_{i j} \partial J_{k l}^{\prime}}=\frac{\beta^{2}}{N} \sqrt{1-t} \sqrt{t}\left(\left\langle s_{i} s_{j} s_{k} s_{l}\right\rangle_{t}-\left\langle s_{i} s_{j}\right\rangle_{t}\left\langle s_{k} s_{l}\right\rangle_{t}\right) .
$$

In general, each derivative with respect to a $J$ or $J^{\prime}$ generates averages of the spins with the indices involved and brings down a prefactor $\beta \sqrt{1-t} / \sqrt{N}$ (for $J$ ) or $\beta \sqrt{t} \sqrt{N}$ (for $J^{\prime}$ ). Fortunately, two derivatives of $\log Z_{t}$ are all we need because when Eq. (A.7) is evaluated, all terms containing higher order derivatives drop out. This is left as an excercise for the reader. Only the following terms survive:

$$
\begin{aligned}
E \frac{\partial}{\partial t} \log Z_{t} \frac{\partial}{\partial \tau} \log Z_{\tau}=\frac{\beta^{4}}{4 N^{2}}\left(2-\frac{\sqrt{1-t} \sqrt{\tau}}{\sqrt{t} \sqrt{1-\tau}}-\frac{\sqrt{1-\tau} \sqrt{t}}{\sqrt{\tau} \sqrt{1-t}}\right) \\
\quad \times E \sum_{i<j, k<l}\left(\left\langle s_{i} s_{j} s_{k} s_{l}\right\rangle_{t}-\left\langle s_{i} s_{j}\right\rangle_{t}\left\langle s_{k} s_{l}\right\rangle_{t}\right)\left(\left\langle s_{i} s_{j} s_{k} s_{l}\right\rangle_{\tau}-\left\langle s_{i} s_{j}\right\rangle_{\tau}\left\langle s_{k} s_{l}\right\rangle_{\tau}\right) \\
+\frac{\beta^{2}}{4 N \sqrt{t \tau}} \sum_{i<j} E\left\langle s_{i} s_{j}\right\rangle_{t}\left\langle s_{i} s_{j}\right\rangle_{\tau} \\
+\frac{\beta^{2}}{4 N \sqrt{1-t} \sqrt{1-\tau}} \sum_{i<j} E\left\langle s_{i} s_{j}\right\rangle_{t}\left\langle s_{i} s_{j}\right\rangle_{\tau} .
\end{aligned}
$$

In this equation, the last term is equal to the penultimate one due to symmetry under the exchange $t \rightarrow 1-t$ and $\tau \rightarrow 1-\tau$. In this equation, we can let the sums run unrestrictedly over $i, j, k, l$ by introducing a factor of $\frac{1}{4}$ for the first sum and a factor of $\frac{1}{2}$ and a correction for the diagonal terms in the second sum, resulting in

$$
\begin{aligned}
E \frac{\partial}{\partial t} \log Z_{t} \frac{\partial}{\partial \tau} \log Z_{\tau}=\frac{\beta^{4}}{16 N^{2}}\left(2-\frac{\sqrt{1-t} \sqrt{\tau}}{\sqrt{t} \sqrt{1-\tau}}-\frac{\sqrt{1-\tau} \sqrt{t}}{\sqrt{\tau} \sqrt{1-t}}\right) \\
\times E \sum_{i j k l}\left(\left\langle s_{i} s_{j} s_{k} s_{l}\right\rangle_{t}-\left\langle s_{i} s_{j}\right\rangle_{t}\left\langle s_{k} s_{l}\right\rangle_{t}\right)\left(\left\langle s_{i} s_{j} s_{k} s_{l}\right\rangle_{\tau}-\left\langle s_{i} s_{j}\right\rangle_{\tau}\left\langle s_{k} s_{l}\right\rangle_{\tau}\right)
\end{aligned}
$$




$$
+\frac{\beta^{2}}{4 N \sqrt{t \tau}}\left(\sum_{i j} E\left\langle s_{i} s_{j}\right\rangle_{t}\left\langle s_{i} s_{j}\right\rangle_{\tau}-N\right) .
$$

We can write $\sum_{i j}\left\langle s_{i} s_{j}\right\rangle_{t}\left\langle s_{i} s_{j}\right\rangle_{\tau}$ as $N^{2}\left\langle\left(\frac{1}{N} \sum_{i} s_{i}^{1 t} s_{i}^{3 \tau}\right)^{2}\right\rangle$, i.e. as a square of the spin overlap

$$
q_{13}(t, \tau)=\frac{1}{N} \sum_{i} s_{i}^{1 t} s_{i}^{3 \tau}
$$

between two replicas labelled 1 and 3 with different interpolation parameters $t$ and $\tau$. The labels 1 and 3 have been chosen because we will shortly need two more replicas which will be assigned the labels 2 and 4 . Replicas 1 and 2 are then understood to have interpolation parameter $t$ and replicas 3 and 4 to have parameter $\tau$. The angular brackets $\langle\cdots\rangle$ without subscript indicate the thermal average of a system comprising independent replicas with interpolation parameters $t$ and $\tau$.

A similar decomposition in replicas can be made in the first part of Eq. (A.11), but here two more replicas are needed. We find

$$
\begin{aligned}
\frac{1}{N^{4}} \sum_{i j k l}\left(\left\langle s_{i} s_{j} s_{k} s_{l}\right\rangle_{t}-\left\langle s_{i} s_{j}\right\rangle_{t}\left\langle s_{k} s_{l}\right\rangle_{t}\right)\left(\left\langle s_{i} s_{j} s_{k} s_{l}\right\rangle_{\tau}-\left\langle s_{i} s_{j}\right\rangle_{\tau}\left\langle s_{k} s_{l}\right\rangle_{\tau}\right) \\
=\frac{1}{N^{4}} \sum_{i j k l}\left(\left\langle s_{i}^{1, t} s_{j}^{1, t} s_{k}^{1, t} s_{l}^{1, t}\right\rangle-\left\langle s_{i}^{1, t} s_{j}^{1, t}\right\rangle\left\langle s_{k}^{2, t} s_{l}^{2, t}\right\rangle\right)\left(\left\langle s_{i}^{3, \tau} s_{j}^{3, \tau} s_{k}^{3, \tau} s_{l}^{3, \tau}\right\rangle-\left\langle s_{i}^{4, \tau} s_{j}^{4, \tau}\right\rangle\left\langle s_{k}^{3, \tau} s_{l}^{3, \tau}\right\rangle\right) \\
=\left\langle\left(q_{13}^{2}-q_{14}^{2}\right)\left(q_{13}^{2}-q_{23}^{2}\right)\right\rangle \\
=\frac{1}{N^{4}}\left\langle\left(\sum_{i j}\left(s_{i}^{1, t} s_{j}^{1, t}-\left\langle s_{i}^{1, t} s_{j}^{1, t}\right\rangle\right)\left(s_{i}^{3, \tau} s_{j}^{3, \tau}-\left\langle s_{i}^{3, \tau} s_{j}^{3, \tau}\right\rangle\right)\right)^{2}\right\rangle \geq 0
\end{aligned}
$$

As a by-product, we see in the last line that the expression $\left\langle\left(q_{13}^{2}-q_{14}^{2}\right)\left(q_{13}^{2}-q_{23}^{2}\right)\right\rangle$ is nonnegative. The end result is finally

$$
\begin{gathered}
E \frac{\partial}{\partial t} \log Z_{t} \frac{\partial}{\partial \tau} \log Z_{\tau}=\frac{N^{2} \beta^{4}}{16}\left(2-\frac{\sqrt{1-t} \sqrt{\tau}}{\sqrt{t} \sqrt{1-\tau}}-\frac{\sqrt{1-\tau} \sqrt{t}}{\sqrt{\tau} \sqrt{1-t}}\right) E\left\langle\left(q_{13}^{2}-q_{14}^{2}\right)\left(q_{13}^{2}-q_{23}^{2}\right)\right\rangle \\
+\frac{N \beta^{2}}{4 \sqrt{t \tau}}\left(E\left\langle q_{13}^{2}\right\rangle-\frac{1}{N}\right) .
\end{gathered}
$$

\section{References}

[1] G. Biroli, J.-P. Bouchaud, and M. Potters. Extreme value problems in random matrix theory and other disordered systems. J. Stat. Mech., 2007:P07019.

[2] D. Sherrington and S. Kirkpatrick. Solvable model of a spin-glass. Phys. Rev. Lett., 35:1792, 1975.

[3] S Cabasino, E Marinari, P Paolucci, and G Parisi. Eigenstates and limit cycles in the SK model. J. Phys. A, 21(22):4201-4210, 1988.

[4] J.-P. Bouchaud, F. Krzakala, and O. C. Martin. Energy exponents and corrections to scaling in Ising spin glasses. Phys. Rev. B, 68:224404, 2003.

[5] M. Palassini. Ground-state energy fluctuations in the Sherrington-Kirkpatrick model. condmat/0307713, 2003.

[6] A. Andreanov, F. Barbieri, and O. C. Martin. Large deviations in spin-glass ground-state energies. Eur. Phys. J. B, 41(3):365-375, 2004.

[7] S. Boettcher. Extremal optimization for Sherrington-Kirkpatrick spin glasses. Eur. Phys. J. B, 46:501-505, 2005.

[8] H. G. Katzgraber, M. Körner, F. Liers, M. Jünger, and A. K. Hartmann. Universality-class dependence of energy distributions in spin glasses. Phys. Rev. B, 72:094421, 2005. 
[9] K. F. Pál. Hysteretic optimization for the Sherrington-Kirkpatrick spin glass. Physica A, 367:261-268, 2006.

[10] T. Aspelmeier, M. A. Moore, and A. P. Young. Interface energies in Ising spin glasses. Phys. Rev. Lett., 90(12):127202, 2003.

[11] A. Crisanti, G. Paladin, H.-J. Sommers, and A. Vulpiani. Replica trick and fluctuations in disordered systems. J. Phys. I France, 2:1325-1332, 1992.

[12] T. Aspelmeier, A. Billoire, E. Marinari, and M. A. Moore. Finite size corrections in the Sherrington-Kirkpatrick model. arXiv:0711.3445v1 [cond-mat.dis-nn], submitted to J. Phys. A, 2007.

[13] J. Wehr and M. Aizenman. Fluctuations of extensive functions of quenched random couplings. J. Stat. Phys., 60(3-4):287, 1990.

[14] T. Aspelmeier and M. A. Moore. Free energy fluctuations in Ising spin glasses. Phys. Rev. Lett., 90(17):177201, 2003.

[15] T. Aspelmeier. Free energy fluctuations and chaos in the Sherrington-Kirkpatrick model. arXiv:0712.3586v1 [cond-mat.dis-nn], accepted by Phys. Rev. Lett., 2007.

[16] T. Aspelmeier. Bond chaos in the Sherrington-Kirkpatrick model. arXiv:????.????, see this mailing, 2008.

[17] M. Mézard, G. Parisi, and M.A. Virasoro. Spin Glass Theory and Beyond. World Scientific, Singapore, 1987.

[18] G. Parisi, F. Ritort, and F. Slanina. Critical finite-size corrections for the SherringtonKirkpatrick spin glass. J. Phys. A, 26:247-259, 1993.

[19] J. Yeo, M. A. Moore, and T. Aspelmeier. Nature of perturbation theory in spin glasses. J. Phys. A, 38(18):4027-4045, 2005.

[20] A. Billoire. Numerical estimate of the finite-size corrections to the free energy of the SherringtonKirkpatrick model using Guerra-Toninelli interpolation. Phys. Rev. B, 73:132201, 2006.

[21] F. Guerra and F. L. Toninelli. The thermodynamic limit in mean field spin glass models. Commun. Math. Phys., 230:71-79, 2002.

[22] G. Parisi and F. Ricci-Tersenghi. On the origin of ultrametricity. J. Phys. A, 33(1):113-129, 2000.

[23] F. Guerra. About the overlap distribution in mean field spin glass models. Int. J. Mod. Phys. $B, 10: 1675,1996$. 